\title{
Tuning the electron transport of molecular junctions by chemically functionalizing anchoring groups: First-principles study
}

\author{
Shigeru Tsukamoto, ${ }^{*}$ Vasile Caciuc, Nicolae Atodiresei, and Stefan Blügel \\ Peter Grünberg Institut and Institute for Advanced Simulation, Forschungszentrum Jülich and JARA, D-52425 Jülich, Germany
}

(Received 3 February 2012; revised manuscript received 4 May 2012; published 20 June 2012)

\begin{abstract}
In this first-principles study, we present density-functional calculations of the electronic structures and electron transport properties of organic molecular junctions with several anchoring groups containing atoms with different electronegativities, i.e., benzenediboronate (BDB), benzenedicarboxylate (BDC), and dinitrobenzene (DNB) molecular junctions sandwiched between two $\mathrm{Cu}(110)$ electrodes. The electronic-structure calculations exhibit a significant difference in the density of states not only at the anchoring groups but also at the aromatic rings of the molecular junctions, suggesting that the electron transport is specific for each system. Our transport calculations show that the BDB and DNB molecular junctions have finite electron transmissions at the zero-bias limit while the BDC molecular junction has a negligible electron transmission. Moreover, for the BDB and DNB systems, the electron transmission channels around the Fermi energy reveal fingerprint features, which provide specific functionalities for the molecular junctions. Therefore, our theoretical results demonstrate the possibility to precisely tune the electron transport properties of molecular junctions by engineering the anchoring groups at the single-atom level.
\end{abstract}

DOI: 10.1103/PhysRevB.85.245435

PACS number(s): 73.23.Ad, 73.63.-b, 85.65.+h

\section{INTRODUCTION}

Since the first suggestion of a molecular nanoscale device, i.e., the single-molecular rectifier designed theoretically by Aviram and Ratner, ${ }^{1}$ molecular electronics exploiting physical and chemical properties of organic molecules has attracted much attention and has been the focus of intense research in the last decade. ${ }^{2}$ In the development of such functional electronic devices built on organic molecules, to understand the electron transport properties of the molecular devices is obviously one of the most important issues to determine device performance.

So far, a number of experimental and theoretical studies on various molecular junctions have been carried out to gain a fundamental understanding of their electron transport properties. ${ }^{3}$ Among many possible choices for organic molecules to be used in molecular devices, the electron transport properties of the benzenedithiolate molecule suspended between a pair of $\mathrm{Au}$ electrodes has been widely studied. ${ }^{4}$ This is mainly motivated by the fact that the strong $\mathrm{S}-\mathrm{Au}$ bond at the hybrid interface, which links the benzene ring to the Au surface, leads to a stable molecule-surface bonding, and such molecular junctions have been extensively studied in experiments. ${ }^{5}$

Recently, the carboxylate group (-COO) has been experimentally and theoretically proposed as an alternative anchoring group that binds benzoate $\left(\mathrm{C}_{6} \mathrm{H}_{5}-\mathrm{COO}\right),{ }^{6}$ monoterephthalate $\left(\mathrm{HOOC}-\mathrm{C}_{6} \mathrm{H}_{4}-\mathrm{COO}\right){ }^{7}$ and pyridine-2-carboxylic-5carboxylate (HOOC- $\left.\mathrm{C}_{5} \mathrm{NH}_{3}-\mathrm{COO}\right)^{8}$ molecules to a $\mathrm{Cu}(110)$ surface via $\mathrm{O}-\mathrm{Cu}$ bonds. Furthermore, a fine tuning of the electric structure of a monoterephthalate molecule adsorbed on a $\mathrm{Cu}(110)$ surface can be achieved by substituting the $\mathrm{C}-\mathrm{H}$ groups in the aromatic ring with $\mathrm{N}$ atoms. ${ }^{9}$

As mentioned above, these studies have been mainly focused on the description of the geometrical and electronic structures of organic molecules adsorbed on a metal surface. However, to integrate the functionality of the carboxylatemetal bond in future molecular electronic devices, it is necessary to investigate in detail the electronic structure and the electron transport properties of such organic molecules bridging between two metal electrodes.

As a first step to reach this goal, an important issue is to establish the relation between a specific chemical structure of the anchoring bond and the electron transport properties of the electrode-molecule-electrode junctions. In the case of molecular junctions with a S-Au anchoring bond, only a few systematic studies performed by Ke et al. ${ }^{10}$ and Di Ventra and Lang ${ }^{11}$ have addressed this problem, by replacing the anchoring $\mathrm{S}$ atoms between the benzene ring and the $\mathrm{Au}$ electrodes with chalcogen atoms and examining the change in the conductance of the molecular junctions. However, for reliable design of molecular electronic devices it is important to understand in detail how the electron transport changes as a function of characteristic chemical structure of the anchoring groups.

Therefore, in this paper, we systematically investigate the change in the electronic structure and electron transmission of three chemically functionalized molecular junctions via different anchoring groups. More precisely, we consider benzenedicarboxylate (OOC- $\mathrm{C}_{6} \mathrm{H}_{4}-\mathrm{COO}, \mathrm{BDC}$ for short, also known as terephthalate), ${ }^{12}$ benzenediboronate (OOB- $\mathrm{C}_{6} \mathrm{H}_{4}-\mathrm{BOO}, \mathrm{BDB}$ for short, also known as phenyldiboronate), ${ }^{13}$ and dinitrobenzene $\left(\mathrm{OON}-\mathrm{C}_{6} \mathrm{H}_{4}-\mathrm{NOO}, \mathrm{DNB} \text { for short }\right)^{14}$ molecules attached to two $\mathrm{Cu}(110)$ electrodes via carboxylate- $\mathrm{Cu}$, boronate- $\mathrm{Cu}$, and nitro- $\mathrm{Cu}$ anchoring bonds, respectively. The choice of these molecular systems is motivated by the observation that, compared to the carboxylate group, in the boronate group the carbon is replaced by a less electronegative B atom, while in the nitro group the carbon is replaced by a more electronegative $\mathrm{N}$ atom. To the best of our knowledge, no studies on the molecular junctions of benzenedicarboxylate, benzenediboronate and dinitrobenzene have been reported so far.

As a consequence, the electronic structures of these molecular junctions are significantly different both at the anchoring groups and at the aromatic ring, suggesting that the electron transport properties can exhibit specific features for each 
system. Indeed, our calculations of ballistic electron transport show clear fingerprints in the electron transmissions around the Fermi energy at zero bias: The BDB and DNB molecular junctions have finite electron transmissions, while the electron transmission of the BDC molecular junction is negligible. Furthermore, for the BDB and DNB molecular junctions the electron transmission spectra significantly differ above the Fermi energy, which differentiate these systems. Moreover, these molecular junctions are also expected to have different electron transport properties when applying finite bias voltages.

This paper is organized as follows. In the next section, we describe the computational methods used in this study and present molecular junction models employed in this work. In Sec. III, we analyze the electronic structures and electron transport properties of the molecular junctions incorporating different anchoring groups, while in Sec. IV we summarize the results obtained in our comparative study.

\section{CALCULATION METHODS AND MODELS}

All calculations presented in this paper are performed within the framework of the density functional theory (DFT). ${ }^{15}$ To determine the electronic ground states of the organic molecular junction systems to be employed in this work, we use an electronic-structure calculation code based on the real-space finite-different formalism, ${ }^{16}$ in which the normconserving pseudopotentials ${ }^{17}$ proposed by Troullier and Martins ${ }^{18}$ are incorporated to describe the interaction between valence electrons and an ion core, and the exchange-correlation interaction is treated by the local density approximation (LDA). ${ }^{19}$ The electronic-structure calculation method enables us to determine the self-consistent electronic ground state with a high degree of accuracy by means of the time-saving double-grid technique. ${ }^{20}$

In the computations of the electron transmissions through the molecular junctions, we employ a code calculating ballistic electron transport based on the real-space finite-difference formalism $^{21}$ in order to maintain consistency with the electronic-structure calculations. The self-consistent potential determined by the electronic structure calculations and pseudopotentials used to calculate it are the input into the electrontransport code, and hence the scattering wave functions of electrons inside the molecular junctions are determined to the given potential non-self-consistently. It has been reported that the non-self-consistent procedure is just as accurate within the linear response regime but significantly more efficient than performing self-consistent expensive computations on a scattering wave basis. ${ }^{22}$ Electron transmission $G(E)$ is evaluated by means of the Landauer-Büttiker formula, ${ }^{23}$ i.e.,

$$
G(E)=G_{0} \sum_{i, j} T_{i j}(E) .
$$

$G_{0}$ is the quantized conductance, and $G_{0}=2 e^{2} / h$, where $e$ is the electron charge and $h$ is Planck's constant. Here, $T_{i j}$ is the transmission probability of electrons flowing from the $i$ th channel in an electrode to the $j$ th channel in another electrode, and is obtained from the scattering wave functions by means of the channel decomposition technique. ${ }^{24}$

It is important to keep in mind that the use of the LDA functional in DFT calculations may result in the overestimation

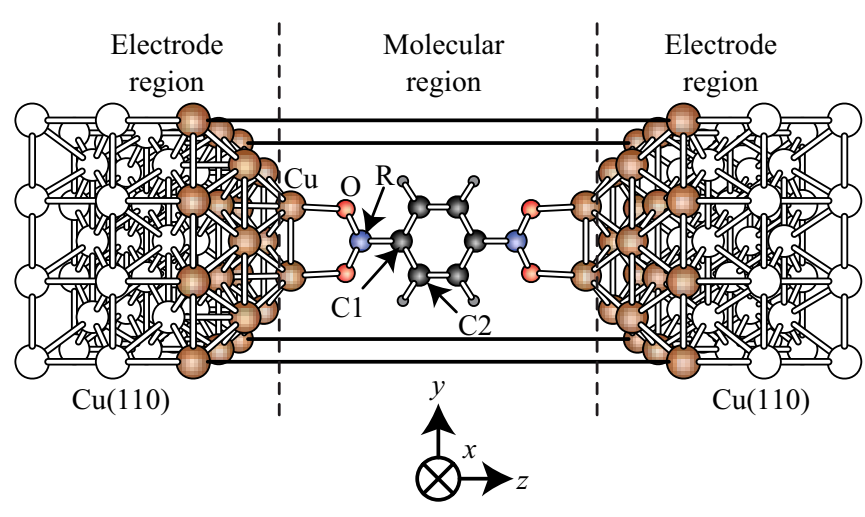

FIG. 1. (Color online) A schematic representation of the molecular junction models employed in this work. To form a junction geometry, a Cu-terminated organic molecule $\left(\mathrm{Cu}_{2}-\mathrm{OOR}-\mathrm{C}_{6} \mathrm{H}_{4}-\mathrm{ROO}\right.$ $\mathrm{Cu}_{2}$ ) defines the molecular region and is suspended between a pair of $\mathrm{Cu}$ electrodes named the electrode regions, where $R$ represents $\mathrm{B}, \mathrm{C}$, and $\mathrm{N}$ for BDB, BDC, and DNB molecules, respectively. Note that the direction $x$ is perpendicular to the molecular plane and the direction $z$ is parallel to the junction direction. The solid lines represent the supercell used in the calculations with periodic boundary conditions. The positions of the colored atoms are relaxed.

of electron transmission because of the underestimation of the band gap. ${ }^{25}$ However, such an approach is well known to give insight into the qualitative trends of electron transport properties. $^{26}$

Figure 1 shows a schematic representation of the computational model employed in this work. All BDB, BDC and DNB molecules are planar, and they differ from each other through one atom in each anchoring group, i.e., a boronate (in BDB) contains a $\mathrm{B}$ atom, a carboxylate (in BDC) contains a $\mathrm{C}$ atom, and a nitro (in DNB) contains a $\mathrm{N}$ atom.

In order to explicitly include the effect of the local chemical bonding between the molecule and the metal electrodes in the molecular junction structures (see Fig. 1) each molecule is sandwiched between a pair of $\mathrm{Cu}(110)$ electrodes with two $\mathrm{Cu}$ adatoms at each end. More exactly, we assume a molecular junction structure such that each organic molecule is attached to a pair of $\mathrm{Cu}$ adatoms lying on the $\mathrm{Cu}(110)$ surface. Besides this, in the most stable adsorption geometry at the interface between the molecule and a $\mathrm{Cu}$ surface, each $\mathrm{O}$ atom in the anchoring groups attaches to a $\mathrm{Cu}$ adatom, ${ }^{6}$ i.e., two strong $\mathrm{O}-\mathrm{Cu}$ bonds are formed at the molecule-substrate interface such that the $\mathrm{O}-\mathrm{O}$ distances in the anchoring groups are almost commensurate with the $\mathrm{Cu}-\mathrm{Cu}$ distance in the [110] direction of a $\mathrm{Cu}(110)$ surface. ${ }^{27}$ Hereafter, the molecule with the $\mathrm{Cu}$ adatoms is referred to as $\mathrm{Cu}$-terminated molecule.

Structural optimization of the molecular junctions has been performed using the following procedure. First, an isolated $\mathrm{Cu}$-terminated molecule is relaxed, and then each end of the relaxed molecule is attached to a $\mathrm{Cu}(110)$ ideal surface in order to form the $\mathrm{Cu}$ fcc crystal structure with a distance of $1.29 \AA$ ( 2.43 bohrs $),{ }^{28}$ as depicted in Fig. 1. Next, the positions of the colored atoms in the junction structure, shown in Fig. 1, are relaxed under the restriction of the three-dimensional periodic boundary conditions, i.e., four bulk-like $\mathrm{Cu}(110)$ layers in each electrode are fixed. 
We have employed another type of molecular-junction system as well, in which the three bulk-like $\mathrm{Cu}(110)$ layers in each electrode are replaced with a jellium model. In other words, each $\mathrm{Cu}$ electrode is represented by three $\mathrm{Cu}(110)$ layers and a following jellium part. In the jellium model, the only parameter needed to be specified is the Wigner-Seitz radius, which is set to $0.64 \AA$ ( 1.21 bohrs). The purpose of using these two junction systems is to see how much the jellium approximation affects the electronic structures around the molecular region (see Fig. 1), which is essential to the transport property. The jellium approximation is just required by the electron transport calculation method used in this work, and allows us to compare the electronic structure and electron transport directly.

\section{RESULTS AND DISCUSSION}

In this section we first discuss in detail the electronic structures of the three molecular junctions considered in our study. Their electronic structures are investigated through projected density of states (PDOS) $N_{\sigma}(E)$ and $N_{\pi}(E)$, where the former and the latter represent PDOS evaluated from the $\sigma$ - and $\pi$-characteristic molecular orbitals, respectively. The PDOS is formulated as

$$
N_{\sigma(\pi)}(E)=\sum_{i} n_{i, \sigma(\pi)} f\left(E-\varepsilon_{i}\right),
$$

where $n_{i, \sigma(\pi)}$ is the atomic population originating from the $i$ th molecular orbital, and $f\left(E-\varepsilon_{i}\right)$ is a broadening function with the center at the eigenenergy of the $i$ th molecular orbital, $\varepsilon_{i}$. In this work, we have chosen a Gaussian function for the broadening function. ${ }^{29}$ The atomic population $n_{i}$ for the molecular-orbital index $i=1,2, \ldots$ is determined by

$$
n_{i, \sigma(\pi)}=\sum_{j \in \sigma(\pi)} c_{i j}^{*} c_{i j}
$$

Here, $c_{i j}$ is the projection coefficient of the $i$ th molecular orbital $\psi_{i}$ onto the $j$ th atomic basis function $\phi_{j}$ as $c_{i j}=$ $\left\langle\phi_{j} \mid \psi_{i}\right\rangle$, and the set of the atomic basis functions $\left\{\phi_{j}\right\}$ is orthonormalized. For this kind of population analysis using the Mulliken formalism, ${ }^{30}$ it is widely accepted that the absolute magnitudes of the atomic populations yielded by the formalism above have little physical meaning, since they still have a high degree of sensitivity depending on the atomic basis set with which one performs the analysis. ${ }^{31}$ However, consideration of their relative values can yield useful information, ${ }^{32}$ as far as a consistent basis set is provided for the population analysis.

Figure 2 shows PDOS with respect to the atomic basis functions of the $\mathrm{Cu}, \mathrm{O}, R(=\mathrm{B}, \mathrm{C}$, and $\mathrm{N})$, and the aromatic ring (referred to as Ring) for each of the Cu-terminated BDB, BDC, and DNB molecules. Since the atoms $\mathrm{C} 1$ and $\mathrm{C} 2$ in the aromatic ring (see Fig. 1) are not equivalent, the PDOS curves drawn in the panels labeled Ring are averaged over the six C atoms so as to be comparable with the other PDOS.

The PDOS of the Cu-terminated BDB molecule is shown in Fig. 2(a). A specific feature of this PDOS is the presence of electronic states in the energy range $[-1.0,-0.5] \mathrm{eV}$ at the $\mathrm{C}$, $\mathrm{O}$, and Ring while at the $\mathrm{B}$ atom these states are absent. This suggests that, within this energy range, the atomic orbitals (a) Benzenediboronate $(\mathrm{BDB})+\mathrm{Cu}$ adatoms

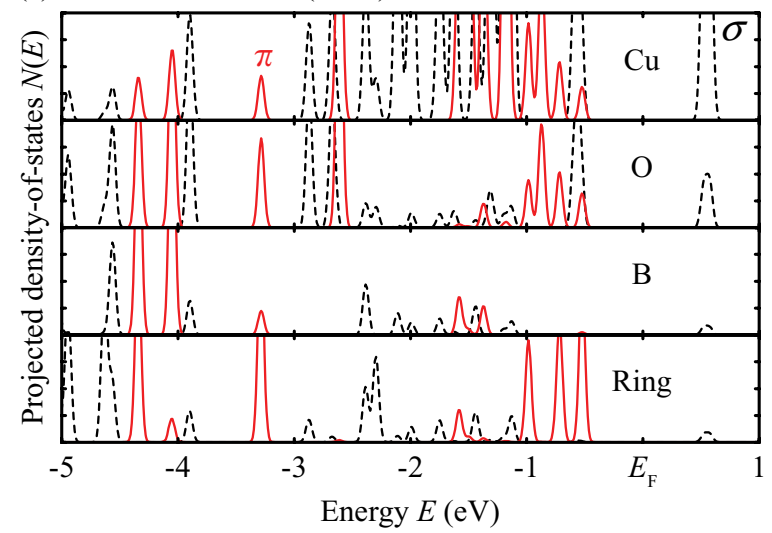

(b) Terephthalate $(\mathrm{BDC})+\mathrm{Cu}$ adatoms

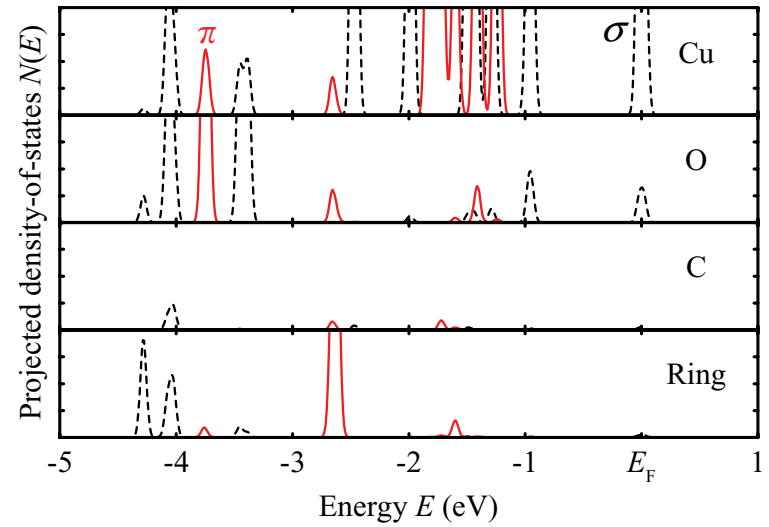

(c) Dinitrobenzene $(\mathrm{DNB})+\mathrm{Cu}$ adatoms

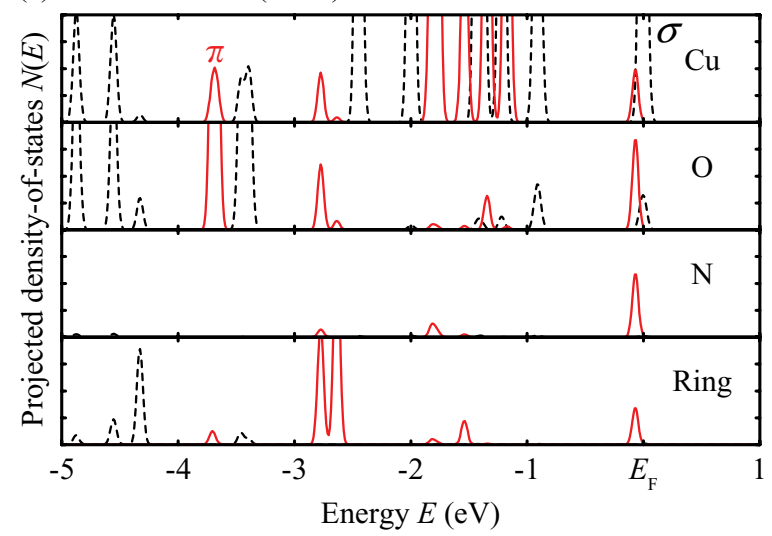

FIG. 2. (Color online) Projected density of states (PDOS) of the $\mathrm{Cu}$-terminated molecules. The panels (a), (b), and (c) correspond to BDB, BDC, and DNB molecular regions defined in Fig. 1. The black dashed and red solid curves represent $\sigma$ and $\pi$ character of the molecular orbitals. The Fermi energy $E_{\mathrm{F}}$ is set at the middle of the highest occupied molecular orbital (HOMO) and the lowest unoccupied molecular orbital (LUMO). For the $\mathrm{Cu}$-terminated BDC and DNB molecules, the PDOS peak of the $\sigma$ character at the Fermi energy $E_{\mathrm{F}}$, which looks like a single peak, contains the HOMO and LUMO with an energy separation of $<40 \mathrm{meV}$.

of the B atom do not contribute to the molecular orbitals which must have node structures at the B atoms. For the other two $\mathrm{Cu}$-terminated molecules, we can see a very small PDOS contributions at the $\mathrm{C}$ and $\mathrm{N}$ atoms over a wider energy range from $-4.0 \mathrm{eV}$ to the Fermi energy $E_{\mathrm{F}}$. Moreover, unlike the 
(a) BDB molecular region $+\mathrm{Cu}$ crystalline electrodes

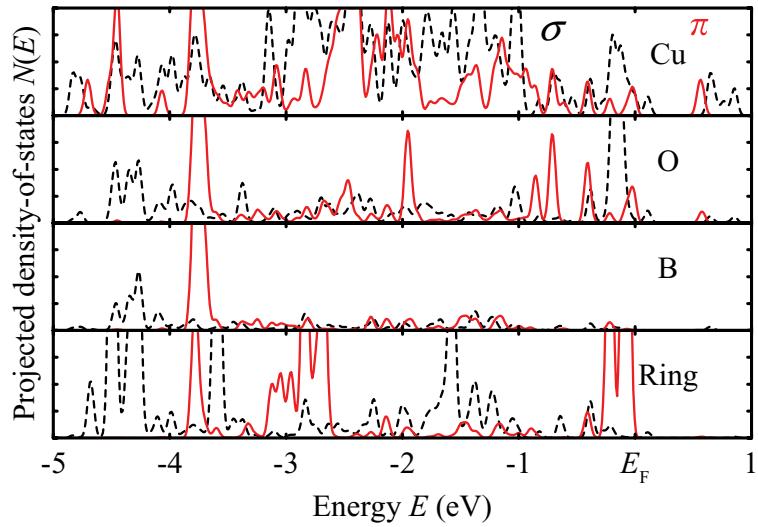

(b) $\mathrm{BDB}$ molecular region $+\mathrm{Cu}$ layers + jellium parts

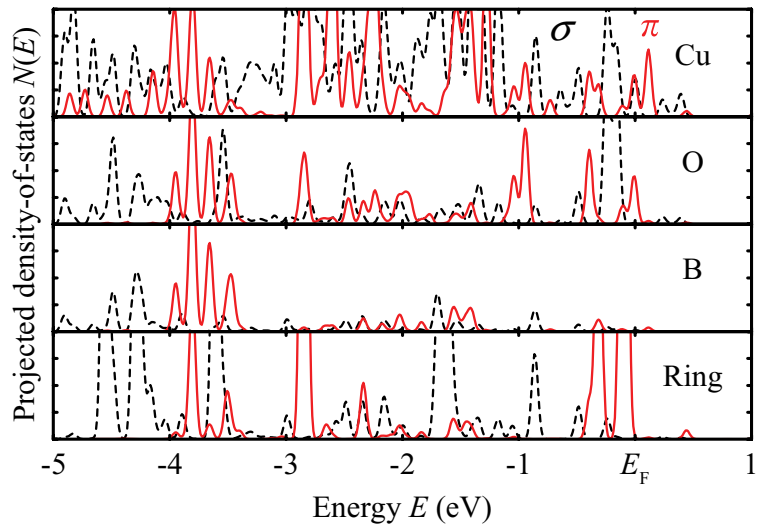

FIG. 3. (Color online) Projected density of states of the BDB molecular junctions. Panels (a) and (b) are for the calculation models without and with the jellium parts, respectively. The black dashed and red solid curves represent $\sigma$ and $\pi$ character of the molecular orbitals.

Cu-terminated BDB molecule, the Cu-terminated BDC and DNB molecules do not have PDOS at their aromatic rings over the energy range from $-1.5 \mathrm{eV}$ to the Fermi energy $E_{\mathrm{F}}$. These differences in the electronic structures are expected to affect the electron transport properties of the molecule in junction structures. In addition, the prominent PDOS peak at the Fermi energy $E_{\mathrm{F}}$, which is commonly observed over all four panels in Fig. 2(c), can contribute to the electron transport properties of the DNB molecular junction as well.

To analyze the electron transport properties of the molecular junctions in more detail, in the following, we move to more realistic molecular junction systems with/without the jellium model as shown in Fig. 1, and examine their electronic structures and the ballistic electron transport properties.

Figures 3, 4, 5(a), and 5(b) compare the PDOS at the molecular regions of the systems with and without the jellium approximation for BDB, BDC, and DNB molecular junctions, respectively. PDOS of the two BDB molecular junctions, shown in Fig. 3, present several common features: Two large PDOS peaks originating from $\pi$ orbitals of the aromatic ring are clearly observed just below the Fermi level $E_{\mathrm{F}}$, small PDOS peaks are seen at the $\mathrm{B}$ atoms, and at the $\mathrm{O}$ atoms one large $\sigma$-orbital peak and three $\pi$-orbital peaks are found below the Fermi level $E_{\mathrm{F}}$. For the BDC molecular junction, by (a) $\mathrm{BDC}$ molecular region $+\mathrm{Cu}$ crystalline electrodes

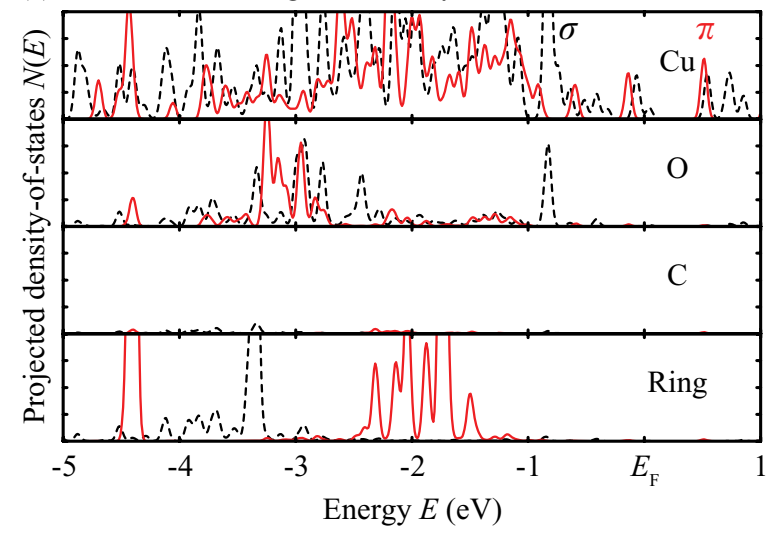

(b) BDC molecular region $+\mathrm{Cu}$ layers + jellium parts

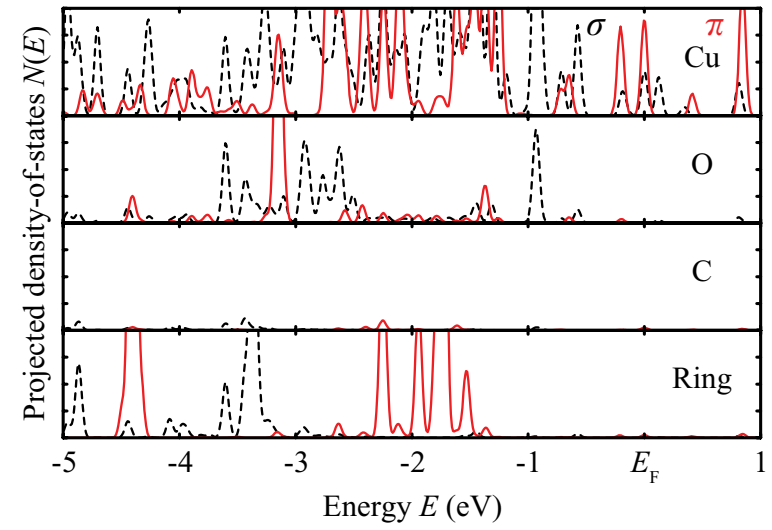

FIG. 4. (Color online) Projected density of states of the BDC molecular junctions. Panels (a) and (b) are for the calculation models without and with the jellium parts, respectively. The black dashed and red solid curves represent $\sigma$ and $\pi$ character of the molecular orbitals.

comparing Figs. 4(a) and 4(b) one can see that the PDOS of the $\mathrm{C}$ atom and the aromatic ring are almost identical, and that the PDOS for the $\mathrm{O}$ atom and the $\mathrm{Cu}$ adatoms do not show a significant difference between the two models. Therefore, it can be concluded that the influence of the jellium approximation on the electronic structure of the molecular region is small enough for the $\mathrm{BDB}$ and $\mathrm{BDC}$ molecular junctions, and hence the electronic structures of the molecular region of these junctions are not disturbed artificially even in the models with the jellium parts.

On the other hand, for the DNB molecular junction, the PDOS peak at the energy of $-0.07 \mathrm{eV}$ in Fig. 5(a) is identified with that at the energy of $-0.46 \mathrm{eV}$ in Fig. 5(b), because these peaks are confirmed to originate from the same molecular state, as seen in Figs. 5(c) and 5(e). In the same way, the PDOS peaks indicated by (d) and (f) are identified with each other as well. This energy shift reveals that the jellium approximation used in our calculations still affects the electronic structures of the molecular region in the case of this molecular junction. The energy shift to the negative side can be attributed to the electronegativity of the $\mathrm{N}$ atoms attracting more electrons than $\mathrm{B}$ and $\mathrm{C}$ atoms. Therefore, in the following discussion on the electron transport through the DNB molecular junction, we have to keep in mind this energy shift. 
(a) DNB molecular region $+\mathrm{Cu}$ crystalline electrodes
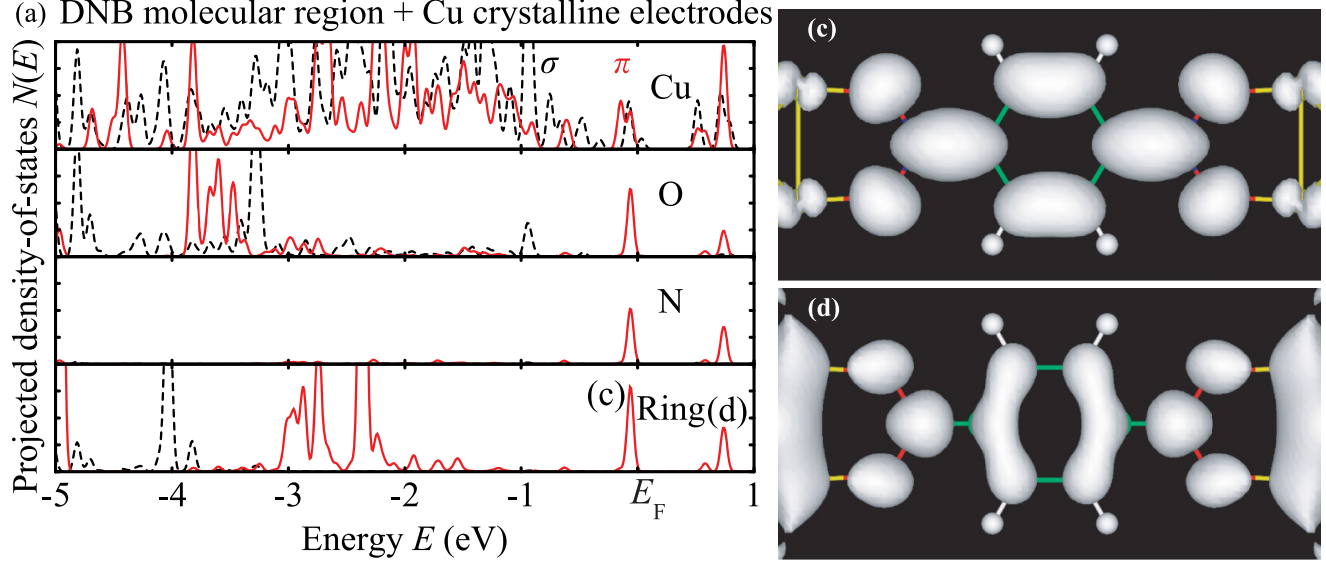

(b) DNB molecular region $+\mathrm{Cu}$ layers + jellium parts

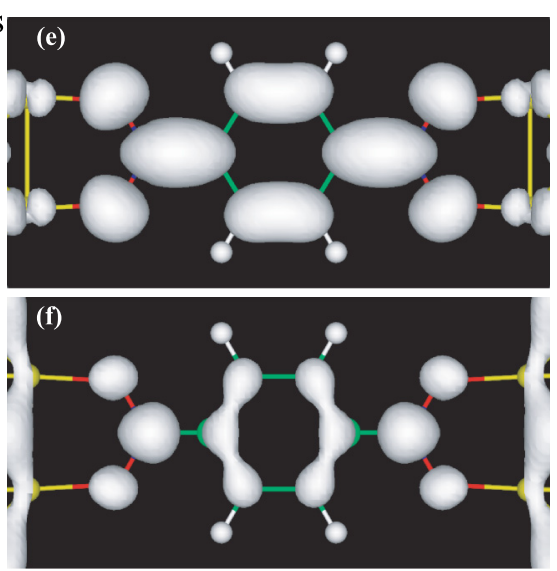

FIG. 5. (Color online) Projected density-of-states (PDOS) of the DNB molecular junctions Panels (a) and (b) are for the calculation models without and with the jellium parts, respectively. Panels (c)-(f) depict the absolute squares of the wave functions corresponding to the PDOS peaks indicated in the panels (a) and (b). The black dashed and red solid curves represent $\sigma$ - and $\pi$-character of the molecular orbitals.

We note that at low binding energies these PDOS peaks preserve the main feature observed in the cases of the $\mathrm{Cu}$ terminated BDB, BDC, and DNB molecules shown in Fig. 2. However, close to the Fermi energy $E_{\mathrm{F}}$ some PDOS peaks at the atoms in the anchoring groups change their energetic position due to the binding of the Cu-terminated molecule to the $\mathrm{Cu}(110)$ electrodes.

Interestingly, in the case of BDC molecular junction, the PDOS of the atoms belonging to the carboxylate groups has a significantly smaller contribution in the energy range $[-1.0$, $+1.0] \mathrm{eV}$ as compared to boronate and nitro anchoring groups. This observation suggests that the BDB and DNB molecular junctions might exhibit a finite conductivity at the zero-bias limit or at small bias voltage.

Moreover, the PDOS indicates that the BDB and DNB molecular junctions are different from each other: In the DNB molecular junction the PDOS peaks around the Fermi energy $E_{\mathrm{F}}$ are present at the $\mathrm{N}$ atoms, while these peaks are suppressed at the $\mathrm{B}$ atoms of the BDB molecular junction, as seen in Figs. 3 and 5. This difference is also expected to lead to a specific electron transport for each of these two molecular junctions.

To compare the electronic structures and the electron transport properties and to discuss the relationship between them, the electron transmissions of the three organic molecular junctions are shown in Fig. 6. As expected from their electronic structures, at zero bias the electron transmissions exhibit a clear difference around and above the Fermi energy $E_{\mathrm{F}}$. Indeed, the BDC molecular junction has no electron transmission at the Fermi energy $E_{\mathrm{F}}$, because of the absence of electronic states at the Fermi energy $E_{\mathrm{F}}$ as shown in Fig. 4(b).

In contrast, the BDB molecular junction has a small electron transmission of $0.02 G_{0}$ at the Fermi energy $E_{\mathrm{F}}$ due to the tail of the transmission peak at $-0.3 \mathrm{eV}$. This transmission peak originates from a hybrid molecular-junction state with a $\pi$ character in the molecular region at the same energy as shown in Fig. 3(b).

The transmission curve for the DNB molecular junction, which does not take into account the energy shift discussed above, exhibits a larger electron transmission of $0.2 G_{0}$ at the Fermi energy $E_{\mathrm{F}}$. When one takes into account the energy shift, the transmission peak at the energy of $-0.45 \mathrm{eV}$ comes close to the Fermi level $E_{\mathrm{F}}$, and hence a much larger transmission value can be expected.

In addition, by analyzing the electron transmission spectra within a small energy range around the Fermi energy, one can also expect nonzero conductivity when applying small bias voltages. At variance with the zero-bias case where no electron transport was present, the current will flow through the BDC molecular junction when applying a bias voltage such that the 


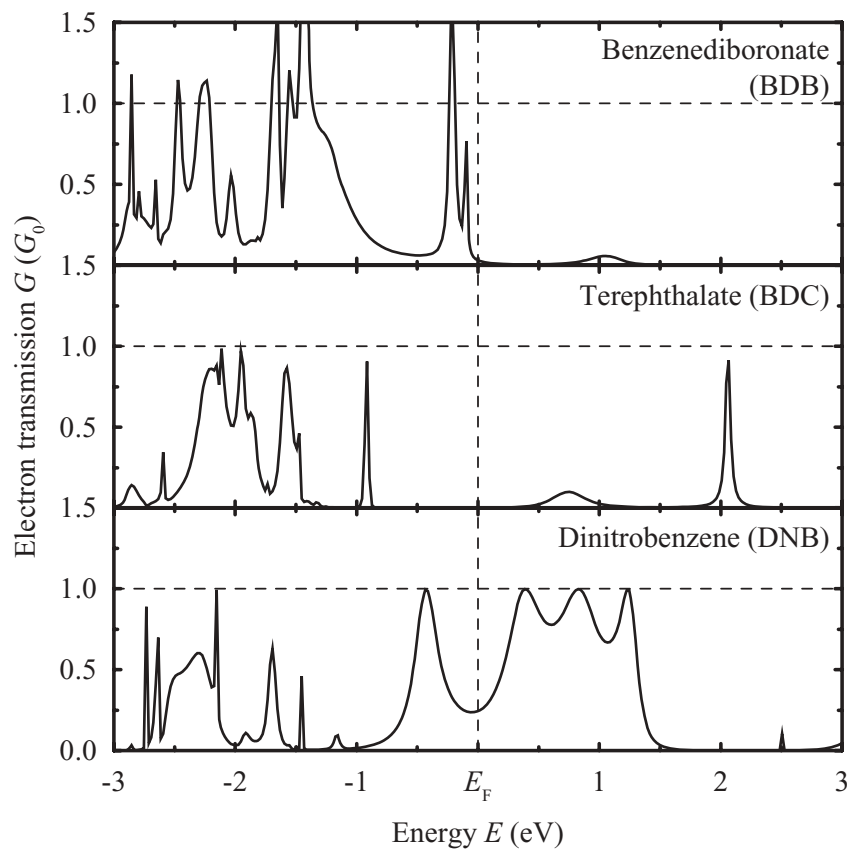

FIG. 6. Electron transmissions $G(E)$ of the BDB, BDC, and DNB molecular junctions as a function of energy of incident electrons. The vertical and horizontal dashed lines represent the Fermi energy $E_{\mathrm{F}}$ and a quantized conductance $1 G_{0}$, respectively. Note that the energy shift in the PDOS of the DNB molecular junction, discussed in the text, is not taken into account.

broad transmission peak at $+0.6 \mathrm{eV}$ will became available for conduction.

For the BDB molecular junction, in addition to the small transmission peak at $+0.8 \mathrm{eV}$, the conductance is expected to significantly increase due to two sharp transmission peaks at the energies around -0.3 and $-0.4 \mathrm{eV}$. In the case of the DNB molecular junction, when applying finite bias voltages the conductivity is expected to change significantly because the valleys and peaks of the transmission curve comes to be involved in the voltage window.

To conclude this section, our first-principles calculations indicate that, for the same molecular core $\left(\mathrm{C}_{6} \mathrm{H}_{4}\right)$, the nitro and boronate anchroring groups will substantially increase the electron transport as compared to the carboxylate one, which exhibits a low conductance. In particular, a low conductance of the metal-carboxylate interface predicted by our calculations agrees well with several electron transport experiments (see, for instance, Ref. 33 and references therein). Therefore, our study clearly demonstrates the possibility of tuning electron transmission by functionalizing the anchoring groups.

\section{CONCLUSION}

To summarize, we have systematically investigated the electronic structures and electron transport properties of the $\mathrm{BDB}, \mathrm{BDC}$, and DNB molecular junctions by changing the chemical nature of one atom in each anchoring group. The chemical functionalization of the anchoring groups was performed starting from the carboxylate group by replacing the $\mathrm{C}$ atom with a less electronegative $\mathrm{B}$ atom (boronate group) and with a more electronegative $\mathrm{N}$ atom (nitro group).

Although the electronic states of the B, C, and $\mathrm{N}$ atoms have a small weight around the Fermi energy, their different electronegativity strongly affects the energetic position of the hybrid states with $\pi$ character at the aromatic ring. As a consequence, the BDB and DNB molecular junctions have a finite PDOS at around the Fermi energy, while the BDC molecular junction has no PDOS at this energy.

This difference is directly reflected in the electron transmission spectra. At zero bias, the former two molecular junctions have finite electron transmissions due to the PDOS peaks present around the Fermi energy, and the latter molecular junction has no electron transmission. However, at small bias-voltage application, the BDC molecular junction will exhibit a small conductance while BDB and DNB molecular junctions will have a significantly large conductance with respect to the zero-bias case.

To conclude, our theoretical results demonstrate the possibility that, by chemical functionalization of the anchoring groups in molecular junctions, a fine tuning of their electron transport properties can be achieved. This is an alternative path to manipulate the conductance of molecular junctions as compared with the possibility of modifying the molecular core. The knowledge gained in our study can be further used to efficiently design future molecular electronic devices with specific functionality.

\section{ACKNOWLEDGMENTS}

The authors acknowledge the help of T. Ono from Osaka University for providing the electronic structure calculation code and for fruitful discussion. This work is supported in part by the Strategic German-Japanese Cooperative Program of Deutsche Forschungsgemeinschaft (DFG) and Japan Science and Technology Agency (JST), and by DFG Priority Program SPP1243. A part of the computations presented in this paper were carried out using the supercomputer JUGENE at Jülich Supercomputing Centre, Forschungszentrum Jülich.
*Corresponding author: s.tsukamoto@fz-juelich.de

${ }^{1}$ A. Aviram and M. A. Ratner, Chem. Phys. Lett. 29, 277 (1974).

${ }^{2}$ C. Joachim, J. K. Gimzewski, and A. Aviram, Nature (London) 408, 541 (2000); A. Nitzan and M. A. Ratner, Science 300, 1384 (2003); L. Lafferentz, F. Ample, H. Yu, S. Hecht, C. Joachim, and L. Grill, ibid. 323, 1193 (2009); J. S. Seldenthuis, F. Prins,
J. M. Thijssen, and H. S. J. van der Zant, ACS Nano 4, 6681 (2010).

${ }^{3}$ Z.-L. Cheng, R. Skouta, H. Vazquez, J. R. Widawsky, S. Schneebeli, W. Chen, M. S. Hybertsen, R. Breslow, and L. Venkataraman, Nature Nanotechnol. 6, 353 (2011); L. Venkataraman, J. E. Klare, C. Nuckolls, M. S. Hybertsen, 
and M. L. Steigerwald, Nature (London) 442, 904 (2006); M. Di Ventra, Electron Transport in Nanoscale Systems (Cambridge University Press, Cambridge, 2008). Y. Imry and R. Landauer, Rev. Mod. Phys. 71, S306 (1999); S. Datta, Electronic Transport in Mesoscopic Systems, edited by H. Ahme, M. Pepper, and A. Broers, Cambridge Studies in Semiconductor Physics and Microelectronic Engineering, Vol. 3 (Cambridge University Press, Cambridge, 1995).

${ }^{4}$ M. A. Reed, C. Zhou, C. J. Muller, T. P. Burgin, and J. M. Tour, Science 278, 252 (1997); M. Di Ventra, S. T. Pantelides, and N. D. Lang, Phys. Rev. Lett. 84, 979 (2000); Y. Kim, T. Pietsch, A. Erbe, W. Belzig, and E. Scheer, Nano Lett. 11, 3734 (2011); D. Q. Andrews, R. P. Van Duyne, and M. A. Ratner, ibid. 8, 1120 (2008); K. Horiguchi, M. Tsutsui, S. Kurokawa, and A. Sakai, Nanotechnology 20, 025204 (2009); J. Nara, W. T. Geng, H. Kino, N. Kobayashi, and T. Ohno, J. Chem. Phys. 121, 6485 (2004); Y. Egami and K. Yamada, Comput. Phys. Commun. 182, 103 (2011); A. Arnold, F. Weigend, and F. Evers, J. Chem. Phys. 126, 174101 (2007).

${ }^{5}$ B. $\mathrm{Xu}$ and N. J. Tao, Science 301, 1221 (2003); X. D. Cui, A. Primak, X. Zarate, J. Tomfohr, O. F. Sankey, A. L. Moore, T. A. Moore, D. Gust, G. Harris, and S. M. Lindsay, ibid. 294, 571 (2001).

${ }^{6}$ M. C. Lennartz, N. Atodiresei, L. Müller-Meskamp, S. Karthäuser, R. Waser, and S. Blügel, Langmuir 25, 856 (2009).

${ }^{7}$ N. Atodiresei, V. Caciuc, K. Schroeder, and S. Blügel, Phys. Rev. B 76, 115433 (2007).

${ }^{8}$ M. C. Lennartz, V. Caciuc, N. Atodiresei, S. Karthäuser, and S. Blügel, Phys. Rev. Lett. 105, 066801 (2010).

${ }^{9}$ V. Caciuc, M. C. Lennartz, N. Atodiresei, S. Karthäuser, and S. Blügel, Nanotechnology 22, 145701 (2011).

${ }^{10}$ S.-H. Ke, H. U. Baranger, and W. Yang, J. Am. Chem. Soc. 126, 15897 (2004).

${ }^{11}$ M. Di Ventra and N. D. Lang, Phys. Rev. B 65, 045402 (2001).

${ }^{12}$ This molecule is already used as a building block of metal-organic framework together with $\mathrm{Cu}$ atoms. For example, see C. Janiak and J. K. Vieth, New J. Chem. 34, 2366 (2010).

${ }^{13}$ This structure can be seen as a part of the benzenediboronic acid molecule, but is not yet isolated. For the benzenediboronic acid molecule, see Boronic Acids: Preparation and Applications in Organic Synthesis and Medicine, edited by D. G. Hall (Wiley-VCH Verlag, Weinheim, 2005).

${ }^{14}$ This molecule has been synthesized and the geometry is already analyzed in the molecular crystal phase. For details, see M. Tonogaki, T. Kawata, S. Ohba, Y. Iwata, and I. Shibuya, Acta Crystallogr. Sect. B 49, 1031 (1993); F. Di Rienzo, A. Domenicano, and L. Riva di Sanseverino, ibid. 36, 586 (1980).
${ }^{15}$ P. Hohenberg and W. Kohn, Phys. Rev. 136, B864 (1964).

${ }^{16} \mathrm{~K}$. Hirose, T. Ono, Y. Fujimoto, and S. Tsukamoto, First-Principles Calculations in Real-Space Formalism (Imperial College Press, London, 2005). T. Ono and K. Hirose, Phys. Rev. B 72, 085115 (2005).

${ }^{17} \mathrm{We}$ have used the norm-conserving pseudopotentials package NCPS97. The details are described in K. Kobayashi, Comput. Mater. Sci. 14, 72 (1999).

${ }^{18}$ N. Troullier and J. L. Martins, Phys. Rev. B 43, 1993 (1991).

${ }^{19}$ J. P. Perdew and A. Zunger, Phys. Rev. B 23, 5048 (1981).

${ }^{20}$ T. Ono and K. Hirose, Phys. Rev. Lett. 82, 5016 (1999).

${ }^{21}$ Y. Egami, K. Hirose, and T. Ono, Phys. Rev. E 82, 056706 (2010); L. Kong, M. L. Tiago, and J. R. Chelikowsky, Phys. Rev. B 73, 195118 (2006); T. Ono, S. Tsukamoto, Y. Egami, and Y. Fujimoto, J. Phys.: Condens. Matter 23, 394203 (2011).

${ }^{22}$ L. Kong, J. R. Chelikowsky, J. B. Neaton, and S. G. Louie, Phys. Rev. B 76, 235422 (2007).

${ }^{23}$ M. Büttiker, Y. Imry, R. Landauer, and S. Pinhas, Phys. Rev. B 31, 6207 (1985).

${ }^{24}$ M. Brandbyge, M. R. Sørensen, and K. W. Jacobsen, Phys. Rev. B 56, 14956 (1997); N. Kobayashi and M. Tsukada, Jpn. J. Appl. Phys. 38, 3805 (1999).

${ }^{25}$ M. Strange, C. Rostgaard, H. Häkkinen, and K. S. Thygesen, Phys. Rev. B 83, 115108 (2011); T. Rangel, A. Ferretti, P. E. Trevisanutto, V. Olevano, and G.-M. Rignanese, ibid. 84, 045426 (2011).

${ }^{26}$ M. Bürkle, J. K. Viljas, D. Vonlanthen, A. Mishchenko, G. Schön, M. Mayor, T. Wandlowski, and F. Pauly, Phys. Rev. B 85, 075417 (2012); D. J. Mowbray, G. Jones, and K. S. Thygesen, J. Chem. Phys. 128, 111103 (2008).

${ }^{27}$ N. Atodiresei, K. Schroeder, and S. Blügel, Phys. Rev. B 75, 115407 (2007); B. G. Frederick, R. J. Cole, J. R. Power, C. C. Perry, Q. Chen, N. V. Richardson, P. Weightman, C. Verdozzi, D. R. Jennison, P. A. Schultz, and M. P. Sears, ibid. 58, 10883 (1998); M. Pascal, C. Lamont, M. Kittel, J. Hoeft, R. Terborg, M. Polcik, J. Kang, R. Toomes, and D. Woodruff, Surf. Sci. 492, 285 (2001).

${ }^{28}$ In this work, the lattice constant of $\mathrm{Cu}$ fcc crystal is set to be $3.64 \AA$ (6.87 bohrs).

${ }^{29} \mathrm{As}$ the broadening function, we have used the Gaussan function, $f(E)=A \exp \left[-(\lambda E)^{2}\right]$ and $\lambda^{-1}=\sqrt{2} k_{B} T$. Here, $A$ is the normalization constant as $A=\lambda / \sqrt{\pi}, k_{B}$ is the Boltzmann constant, and $T$ is temperature set to be $300 \mathrm{~K}$ in this work.

${ }^{30}$ R. S. Mulliken, J. Chem. Phys. 23, 1833 (1955); R. Hoffmann, Rev. Mod. Phys. 60, 601 (1988).

${ }^{31}$ E. R. Davidson and S. Chakravorty, Theor. Chim. Acta 83, 319 (1992).

${ }^{32}$ M. D. Segall, R. Shah, C. J. Pickard, and M. C. Payne, Phys. Rev. B 54, 16317 (1996).

${ }^{33}$ S. Karthäuser, J. Phys.: Condens. Matter 23, 013001 (2011). 